\title{
An unexpected case of pellagra
}

\section{Alicia M Thornton \\ MBBS(Hons), BSC}

Dermatology Registrar

\section{Catherine J Drummond}

MBBS(Hons), FACD

Dermatologist

Department of Dermatology, Canberra Hospital, Canberra, ACT.

aliciathrntn@gmail.com

doi: 10.5694/mjal3.11187

\section{Clinical record}

An anxious 50-year-old woman presented with recurrence of a painful, well demarcated violaceous eruption with erythematous margins and large areas of blistering on her ankles and dorsa of her feet (Box 1, A). She had an erythematous desquamating rash on her hands, forearms and periorbital skin, with surrounding hyperpigmentation (Box 1, B). There were no signs of chronic liver disease, arterial insufficiency or sensory deficit. Oral antibiotics and high-dose oral prednisolone had been ineffective. She had no history of trauma or previous skin conditions. Sun exposure was an aggravating factor. She had recently started taking a herbal diuretic tablet and occasionally used ibuprofen and paracetamol for headaches. Her sister had an undifferentiated connective tissue disease and a positive antinuclear antibody level. The patient was a smoker and reported drinking half a bottle of gin, amounting to at least 10 standard alcoholic drinks, per day.

The differential diagnoses we considered were infection, pseudoporphyria, phototoxic reaction caused by the herbal diuretic tablet or ibuprofen, bullous lupus and porphyria cutanea tarda. Directed investigations (Box 2) did not provide a diagnosis. Mild elevation of some urinary and faecal porphyrins were consistent with liver dysfunction, rather than confirming porphyria. Histopathological examination showed paucicellular interface dermatitis with subepidermal blistering and epidermal pallor. Direct immunofluorescence for immunoglobulin and complement proteins was negative.
All parameters that were outside reference intervals, except mean cell volume and $\gamma$-glutamyl transferase level, returned to normal within 2 weeks of initial management.

Initial supportive management, oral cephalexin 500 mg four times daily, and tapering prednisolone were ineffective. Further history-taking revealed poor nutritional intake, purging using docusate sodium tablets for laxative effect, and intermittent diarrhoea. The patient reported restricting her diet for weight control. She was underweight, with a body mass index of $17 \mathrm{~kg} / \mathrm{m}^{2}$. A scaly hyperpigmented collarette on her upper chest (Box 1, C) resembled Casal necklace, suggesting the clinical diagnosis of vitamin $\mathrm{B}_{3}$ deficiency.

Administering oral nicotinamide $50 \mathrm{mg}$ twice daily resulted in significant improvement in tenderness, burning discomfort and erythema within 2 days, and desquamation within 1 week. After 3 months of supplementation, only mild postinflammatory hyperpigmentation remained, subjective skin sensation had returned, mood had improved and diarrhoea had abated. The patient declined further investigations, including colonoscopy for other potential causes of malabsorption. The eventual resolution of her gastrointestinal symptoms suggested they were due to the disease process rather than a secondary cause such as colitis. She also declined referral to psychology, psychiatry or drug and alcohol counselling but accepted advice from a dietitian. ellagra is caused by a cellular deficiency of niacin or its precursor amino acid, tryptophan. Niacin, also known as nicotinic acid or vitamin $B_{3^{\prime}}$ is essential for carbohydrate, fat, protein and alcohol metabolism, detoxification of drugs and reactive oxygen species, cell signalling and DNA repair. ${ }^{1,2}$

Early gastrointestinal and neurological symptoms are usually subtle and non-specific but if untreated can progress to death from multiorgan failure. Therefore, mucocutaneous signs provide important diagnostic clues. An acute, symmetrical, well demarcated, tender erythematous eruption occurs at sites of sun exposure, heat, friction and pressure. Exacerbations involving the face, neck, chest and dorsa of extremities occur in spring and summer. ${ }^{1-3}$ The eruption can mimic sunburn with vesicles and bullae, termed wet pellagra. ${ }^{4}$ As in this patient, pemphigus pellagrosus with exudative bullae, pruritus and burning may occur on solar re-exposure. ${ }^{2}$ In chronic

\section{The patient's skin lesions}
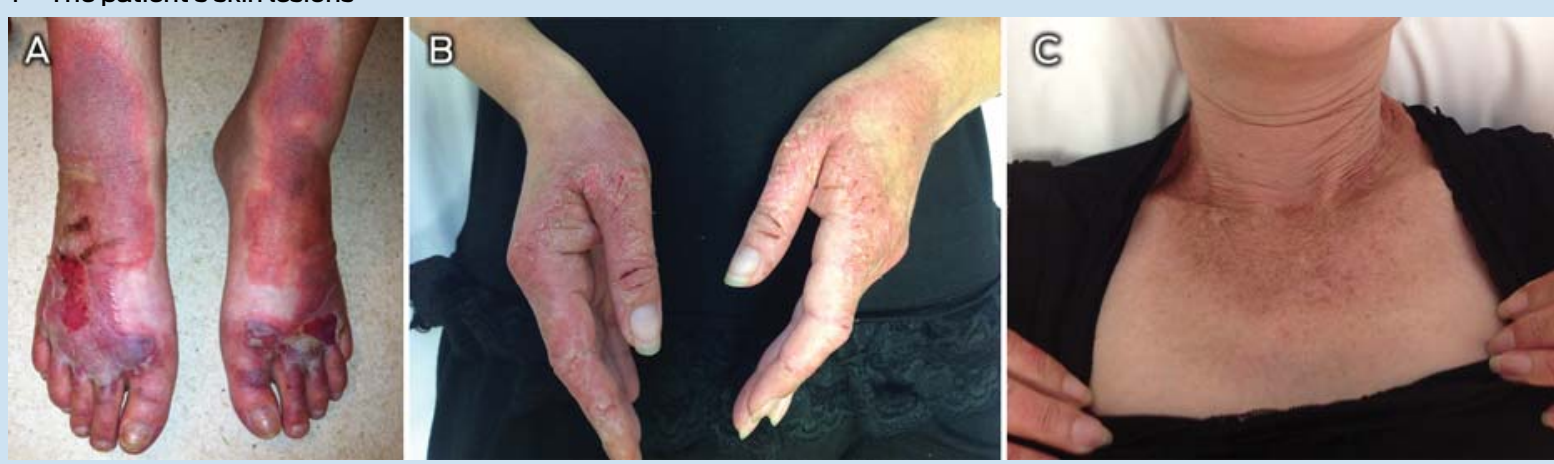

A: Well demarcated violaceous eruption with erythematous margins and large areas of blistering. B: Erythematous desquamating rash, with surrounding hyperpigmentation. C: Scaly hyperpigmented collarette on her upper chest, resembling Casal necklace. 
cases the skin is hyperpigmented and a cinnamon-brown colour. Hyperkeratosis and desquamation produce a dry, rough, scaly or cracked appearance.

Primary pellagra occurs in dietary deficiency of tryptophan or niacin. Secondary pellagra is caused by conditions characterised by interference with absorption or metabolism of tryptophan and niacin, including anorexia nervosa, chronic alcoholism, prolonged diarrhoea, ileitis, colitis, cirrhosis, carcinoid syndrome, Hartnup disease and HIV. Medicines including azathioprine, 5-flurouracil, 6-mercaptopurine and phenobarbitone inhibit the conversion of tryptophan to niacin. Isoniazid is an analogue of niacin and suppresses endogenous formation. ${ }^{1,2}$

Clinical diagnosis is confirmed by rapid response to oral nicotinamide when up to $500 \mathrm{mg}$ daily in divided doses is given. Nicotinamide is the preferred supplement, as niacin causes flushing and headaches. , $3,5^{2}$

Isolated cases of pellagra still occur in developed countries despite dietary fortification and the accessibility of niacin-rich foods. There are no known published reports from Australia. A retrospective study in a hospital population in Spain found the annual incidence was 0.5 cases per 100000 population, ${ }^{3}$ and the most common risk factors for pellagra were alcoholism and poor diet. As in our patient, the diagnosis is often delayed, possibly owing to the rarity of pellagra. 3,6

Factors contributing to alcohol-induced pellagra include poor dietary intake, niacin depletion due to alcohol metabolism, malabsorption due to the toxic effect of alcohol on the gut, and cirrhosis. ${ }^{5}$ Of note, patients with severe psychiatric and neurological forms of the disease such as pellagrous encephalopathy and pellagra psychosis may present without cutaneous signs. The incidence reported in the Spanish study may have underestimated true incidence, as their diagnostic criteria included dermatological manifestations. ${ }^{3}$ One study of 20 cases has suggested that lack of cutaneous involvement in chronic alcoholics with neuropsychiatric symptoms may be due to negligible sun exposure, both in social life and in an acute care setting. ${ }^{6}$ Severe neurological symptoms may progress so quickly that cutaneous signs do not have time to manifest. ${ }^{5}$ The important lesson from these reports is that niacin deficiency is common in chronic alcoholism, and pellagra should be considered a differential diagnosis of delirium tremens and psychosis, especially if signs of gait disturbance, limb rigidity and hyperreflexia are present. $^{5-8}$

Associations with anorexia nervosa and medication interactions are also important. In patients with anorexia nervosa, signs of pellagra may be atypical and overlap with other nutritional deficiencies. ${ }^{9}$ These features may be an ominous sign, as demonstrated by a recent fatality. ${ }^{10}$ Further, isoniazid, azathioprine and antiepileptic drugs have been implicated recently. 3,11,12 Our literature search revealed no reports of pellagra being caused by excessive use of docusate sodium, but this may have contributed to the disease in our patient, owing to malabsorption.

The purpose of this review is to draw attention to the presence of nutritional deficiencies such as pellagra in the developed world. Our patient did not present initially with obvious signs of poor self-care, alcoholism, anorexia
2 Results of initial laboratory tests

Laboratory test

Result

Reference interval (RI)

Microscopy, culture and sensitivity

C-reactive protein

Skin flora cultured, no fungal organisms

$5.3 \mathrm{mg} / \mathrm{L}$

$<5.0 \mathrm{mg} / \mathrm{L}$

Erythrocyte sedimentation rate

$32 \mathrm{~mm} / \mathrm{h}$

$1-30 \mathrm{~mm} / \mathrm{h}$

White blood cells

$19.6 \times 10^{9} / \mathrm{L}$

$4.0-11.0 \times 10^{9} / \mathrm{L}$

Lymphocytes

$0.16 \times 10 \% / L$

$1.2-4.0 \times 10^{9} / \mathrm{L}$

Neutrophils

$17.88 \times 10^{9} / \mathrm{L}$

$1.8-7.5 \times 10^{9} / \mathrm{L}$

Monocytes

$1.57 \times 10^{9} / \mathrm{L}$

$0.10-1.0 \times 10^{9} / \mathrm{L}$

Haemoglobin

$132 \mathrm{~g} / \mathrm{L}$

$115-160 \mathrm{~g} / \mathrm{L}$

Mean cell volume

$112 \mathrm{fL}$

90-96fL

Mean cell haemoglobin

$37.9 \mathrm{pg} / \mathrm{cell}$

27.0-33.0 pg/cell

Red cell distribution width

$14.1 \%$

$11.0 \%-14.5 \%$

Platelets

$278 \times 10^{9} / \mathrm{L}$

$150-400 \times 10 \% / \mathrm{L}$

Creatinine

$65 \mu \mathrm{mol} / \mathrm{L}$

$40-90 \mu \mathrm{mol} / \mathrm{L}$

Urea

$4.6 \mathrm{mmol} / \mathrm{L}$

$2.5-7.5 \mathrm{mmol} / \mathrm{L}$

Bilirubin

$14 \mathrm{\mu mol} / \mathrm{L}$

2-20 $\mathrm{\mu mol} / \mathrm{L}$

Alanine aminotransferase

Aspartate aminotransferase

$121 \mathrm{U} / \mathrm{L}$

$<55 \mathrm{U} / \mathrm{L}$

$118 \mathrm{U} / \mathrm{L}$

5-50U/L

$\gamma$-glutamyl transferase

219U/L

9-36U/L

Lactate dehydrogenase

$285 \mathrm{U} / \mathrm{L}$

$125-240 \mathrm{U} / \mathrm{L}$

Erythrocyte porphyrins

Not elevated

Antinuclear antibody

Not detected

$3 \mathrm{IU} / \mathrm{mL}$

$<40 \mathrm{IU} / \mathrm{mL}$

Double-stranded DNA antibody

HIV-1/2 antigen and antibody

Not detected

Hepatitis B surface antigen

Not detected

Hepatitis C virus antibody

Not detected

IgA tissue transglutaminase antibody

$<3 \mathrm{AU} / \mathrm{mL}$

$<20 \mathrm{AU} / \mathrm{mL}$

Nutritional panel*

Within RIs

Vitamin $\mathrm{B}_{12}$

$956 \mathrm{pmol} / \mathrm{L}$

$138-652 \mathrm{pmol} / \mathrm{L}$

\section{Lessons from practice}

- Pellagra, or niacin (vitamin $B_{3}$ ) deficiency, is a potentially fatal disease which is now rare in the developed world.

- Diagnosis is often delayed owing to non-specific symptoms and low clinical suspicion. The classic triad of dermatitis, diarrhoea and dementia is not always present.

- High-risk groups include those with chronic alcohol dependence, anorexia nervosa and those using medicines such as antiepileptics, azathioprine and isoniazid. Treatment involves nicotinamide supplementation plus redressing these secondary causes and comorbid nutritional deficiencies.

- Awareness of pellagra as a disease entity in the developed world and a clinical suspicion based on knowledge of at-risk populations are key to early diagnosis and prevention of morbidity and mortality.

nervosa or body-image issues. The diagnosis was only made after clinical suspicion led to a careful social and dietary history-taking and examination. Thus, awareness of the condition and of high-risk populations can prevent delayed diagnosis and treatment, with its associated morbidity and mortality.

Competing interests: No relevant disclosures. 
1 Wan P, Moat S, Anstey A. Pellagra: a review with emphasis on photosensitivity. Br J Dermatol 2011; 164: 1188-1200.

2 Hegyi J, Schwartz RA, Hegyi V. Pellagra: dermatitis, dementia, and diarrhoea. Int J Dermatol 2004; 43: 1-5.

3 Piqué-Duran E, Pérez-Cejudo JA, Cameselle D, et al. [Pellagra: a clinical, histopathological, and epidemiological study of 7 cases] [Spanish]. Actas Dermosifiliogr 2012; 103: 51-58.

4 Cho S, Choi JC, Choi JH, et al. Wet pellagra. Int J Dermatol 2001; 40: 543-544.

5 Oldham MA, Ivkovic A. Pellagrous encephalopathy presenting as alcohol withdrawal delirium: a case series and literature review. Addict Sci Clin Pract 2012; 7: 12.

6 Ishii N, Nishihara Y. Pellagra among chronic alcoholics: clinical and pathological study of 20 necropsy cases. J Neurol Neurosurg Psychiatry 1981; 44: 209-215.
7 López M, Olivares JM, Berrios GE. Pellagra encephalopathy in the context of alcoholism: review and case report. Alcohol Alcohol 2013; 7 Aug [Epub ahead of print].

8 Sharma B, Sannegowda RB, Jain R, et al. A rare case of alcoholic pellagra encephalopathy with startle myoclonus and marked response to niacin therapy: time for a new dictum? BMJ Case Rep 2013; 22 Apr. doi: 10.1136/ bcr-2013-008906.

9 Jagielska G, Tomaszewicz-Libudzic EC, Brzozowska A. Pellagra: a rare complication of anorexia nervosa. Eur Child Adolesc Psychiatry 2007; 16: 417-420.

10 Sato M, Matsumura Y, Kojima A, et al. Pellagra-like erythema on sunexposed skin of patients with anorexia nervosa. J Dermatol 2011; 38: 1037-1040.

11 Bilgili SG, Karadag AS, Calka O, Altun F. Isoniazid-induced pellagra. Cutan Ocul Toxicol 2011; 30: 317-319.

12 Oliveira A, Sanches M, Selores M. Azathioprine-induced pellagra. J Dermatol 2011; 38: 1035-1037. 